\title{
NOUVELle
}

\section{Rôle de la phagocytose associée à la protéine LC3 au cours de l'infection par Listeria monocytogenes}

Léa Dutour ${ }^{1}$, દ́léonore Mandonnet ${ }^{1}$, Oliver Nüsse ${ }^{2}$

\author{
${ }^{1}$ MI Biologie Santé, Université Paris-Saclay, 91405 Orsay, \\ France. \\ ${ }^{2}$ Université Paris-Saclay, CNRS, Institut de chimie physique, \\ UMR8000, 91405 Orsay, France. \\ lea.dutour@dbmail.com \\ eleonore.mandonnet@u-psud.fr \\ oliver.nusse@universite-paris-saclay.fr
}

> Listeria monocytogenes, bactérie Grampositive, est à l'origine de la listériose, infection provoquant chez l'homme des septicémies ou des infections du système nerveux central. Cette bactérie est transmise principalement par l'alimentation et infecte plusieurs types cellulaires grâce à sa capacité à franchir les barrières hémato-encéphalique, intestinale et placentaire [1]. Bien que la listériose soit rare, la mortalité associée ainsi que les séquelles neurologiques sont élevées (25 à $30 \%$ ). De précédentes études ont permis de mettre en évidence la capacité de cette bactérie à infecter des cellules immunitaires de l'hôte, les macrophages, et à détruire les membranes des phagosomes par la sécrétion de toxines. Toutefois, dans un article récent de Gluschko et al. [2], il a été montré que l'organisme est capable de combattre l'infection par L. monocytogenes en utilisant une forme particulière de phagocytose associée à la protéine LC3 (microtubule-associated protein IA/1Blight chain 3), la LAP (pour LC3-associated phagocytosis), qui va utiliser certains éléments de la machinerie autophagique. Elle est qualifiée d'autophagie non canonique.

\section{Listeria est internalisée via la LAP}

Dans un premier temps, Gluschko et al. ont voulu caractériser le type d'autophagie (canonique ou LAP) déclenchée par l'infection par L. monocytogenes. Afin de répondre à cette question, ils ont étudié la surface des phagosomes après infection de macrophages par des souches de L. monocytogenes de type sauvage ou déficientes pour différentes fonctions. Ils ont ainsi pu observer, en microscopie confocale, la présence de LC3-II (résultant de la conversion de la forme cytoplasmique LC3-I, en la forme LC3-II associée au phagosome) à la surface des phagosomes ayant internalisé Listeria. Ce recrutement de LC3 dépend de la production d'espèces réactives de l'oxygène (ROS) par la NADPH oxydase (Nox2). Par ailleurs, une analyse structurale, par microscopie électronique à transmission, de la membrane du phagosome contenant L. monocytogenes a permis de mettre en évidence le fait que la majorité des bactéries internalisées sont entourées d'une membrane unique et non d'une double membrane, comme c'est le cas pour l'autophagie canonique. L'ensemble de ces résultats a permis de consolider l'hypothèse selon laquelle la bactérie L. monocytogenes est principalement internalisée par la LAP, un mécanisme d'autophagie caractérisé par le recrutement de LC3 à la membrane des phagosomes, la production de ROS et la présence d'une membrane unique [3].

\section{Mac-1, élément indispensable}

\section{au processus de la LAP}

De précédentes études ayant mis en évidence le rôle essentiel de l'activation de Nox2 lors du recrutement de LC3-II à la membrane du phagosome [4], les auteurs ont étudié le mécanisme d'activation de Nox2 en se concentrant sur l'intégrine $\beta_{2}$ Mac-1 $\left(\alpha_{M} \beta_{2}\right)$. Mac-1 qui fonctionne à la fois comme une molécule d'adhérence et comme un récepteur de certains ligands bactériens, est exprimé à la surface des macrophages [5] et est constitué des sous-unités $\operatorname{CD} 18\left(\beta_{2}\right)$ et $\operatorname{CDllb}\left(\alpha_{M}\right)$. Macl reconnaît et interagit avec $L$. monocytogenes. La production de ROS par les macrophages infectés est significativement diminuée dans des souris déficientes pour la sous-unité CDllb. Cette diminution de la production de ROS est également associée à un moindre recrutement de LC3 dans les phagosomes contenant la bactérie pathogène. Zhang et al. avaient démontré en 2008 [6] que la production de ROS était dépendante de l'activation de Nox2, résultant elle-même du rapprochement des différentes sous-unités de Nox2 à la membrane plasmique dans des zones membranaires enrichies en céramides. II est important de noter que ces zones sont créées par l'action de la sphingomyélinase acide (ASMase), dégradant la sphingomyéline en céramides [7]. Soulignons le fait qu'une déficience de la sous-unité CDllb de Mac-l dans les macrophages provoque une diminution de l'activité d'ASMase, qui se traduit par une baisse de la production de ROS. Cela permet de conclure que, dans ces conditions, l'activation de Nox2 dépend de celle de l'ASMase, elle-même dépendante de Mac-1. Les auteurs ont pu mettre en évidence, par différents systèmes expérimentaux, l'existence d'une boucle de rétroaction positive entre Nox2 et ASMase, activée par Mac-1, en invalidant le gène de Nox2 (souris Nox2-/-), ou en inhibant spécifiquement Nox2 avec l'inhibiteur spécifique VAS-2780, ou encore en piégeant les ROS produits par Nox2 avec de la $\mathrm{N}$-acétylcystéine. Ils ont ainsi mis en évidence le fait que les ROS produits amplifient l'activité ASMase, qui permettra ainsi alors de fournir une production optimale de ROS. L'ASMase est donc aussi impliquée, via Nox2, dans le recrutement de LC3-II sur le phagosome (Figure 1). 


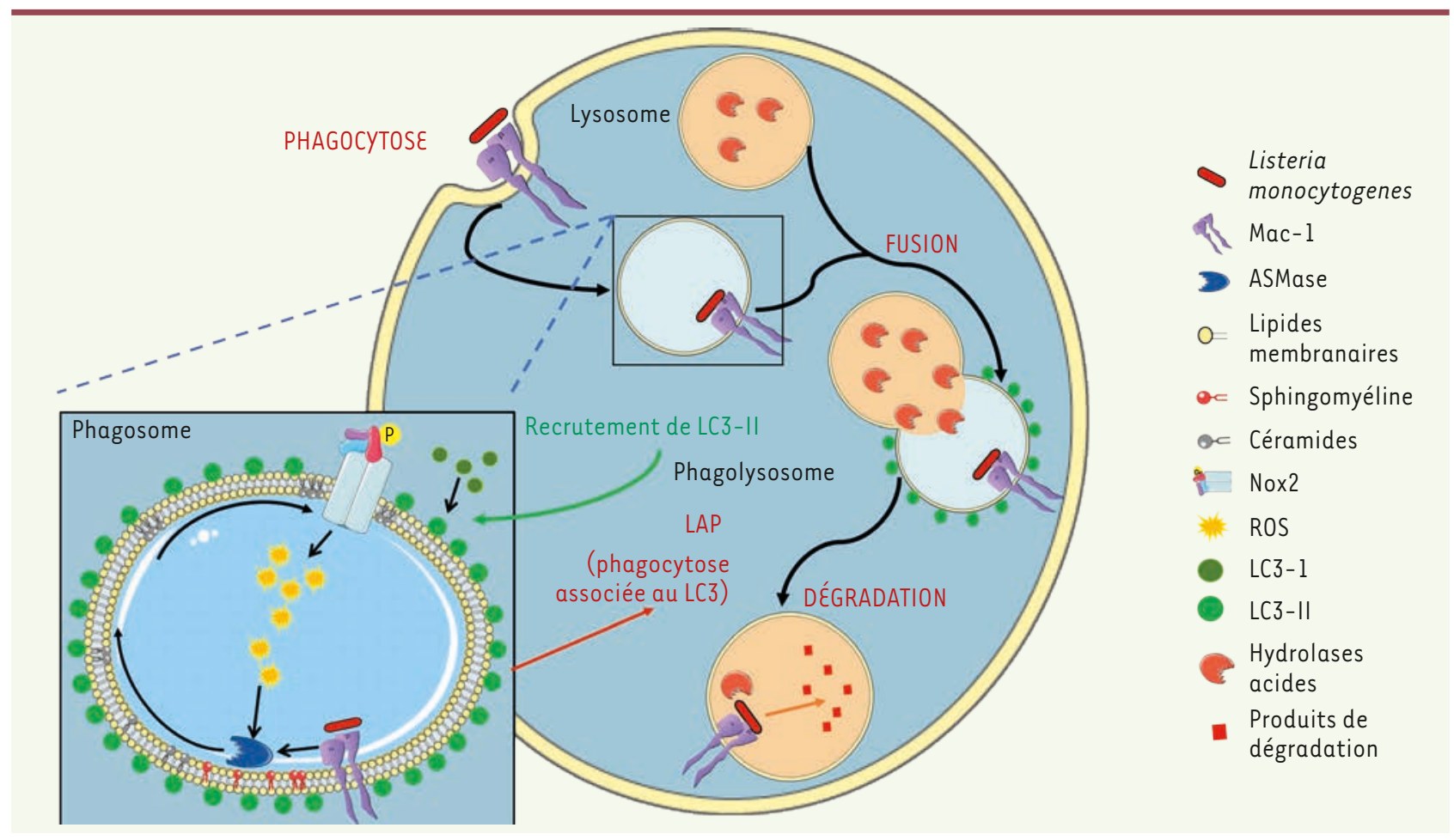

Figure 1. Mécanisme de la phagocytose associée au LC3 lors d'une infection par la bactérie L. monocytogenes. La bactérie se fixe sur le récepteur Mac-1, activant la conversion par l'ASMase des sphingomyélines en céramides et entrainnant la production de ROS par Nox2. Cela permet le recrutement de LC3 sur le phagosome, et favorise ainsi la fusion du phagosome et du lysosome, suivie de la dégradation de la bactérie par les hydrolases acides.

\section{L'élimination de $L$. monocytogenes se fait par la LAP}

L'ASMase a un rôle indispensable lors de la fusion des lysosomes avec les phagosomes [8]. La fusion de ces deux compartiments est nécessaire pour la dégradation de $L$. monocytogenes par les hydrolases acides des lysosomes, ce qui contribue à l'immunité anti-listeria [9]. Gluschko et al. ont étudié la possibilité que LC3, en aval de l'ASMase, puisse favoriser la fusion des lysosomes avec les phagosomes contenant L. monocytogenes et permettre ainsi la dégradation de la bactérie. Pour cela, ils se sont intéressés au transfert de la cargaison lysosomale lors de la fusion d'un lysosome avec un phagosome LC $3^{+}$. En préchargeant des lysosomes avec des billes de latex fluorescentes, ils ont pu établir une corrélation entre la présence de LC3 sur le phagosome et l'acquisition de la cargaison lysosomale. De surcroît, lorsque les macrophages des souris sont déficients en Atg7, une protéine activatrice de l'autophagie, ou en Nox2, il n'y a pas de conversion de LC3-I en LC3-II, forme impliquée dans la fusion du phagosome, ni de recrutement de LC3-II sur les phagosomes contenant la bactérie. Les auteurs observent donc ici une réduction de la colocalisation entre L. monocytogenes et la cargaison lysosomale dans le phagolysosome. L'ensemble de ces observations met en évidence le fait que l'autophagie de type LAP permet d'augmenter l'exposition de L. monocytogenes aux hydrolases acides des lysosomes en favorisant la fusion, via la présence de LC3-II, des phagosomes contenant $L$. monocytogenes avec les lysosomes [10]. À la suite de cette fusion favorisée par la LAP, les hydrolases acides lysosomales vont pouvoir venir dégrader la bactérie pathogène et ainsi contrôler l'infection de manière efficace. Dans ce processus de mise en place d'une immunité anti-listeria, seule l'autophagie non canonique (LAP), via son induction par Mac-1, participe efficacement à l'élimination de L. monocytogenes par les macrophages. La LAP va favoriser la réponse immunitaire anti-listeria au détriment de la capacité du pathogène à persister dans ces cellules.

\section{Le début de la fin pour la Listeria?}

Gluschko et al. ont donc montré que lors d'une infection par la bactérie L. monocytogenes, la cellule hôte détruit ce pathogène par un mécanisme d'autophagie non canonique, appelé LAP. La caractérisation des mécanismes impliqués dans la réponse immunitaire anti-listeria a révélé l'importance de la production de ROS par activation de Nox2 et de l'ASMase, toutes deux dépendantes de Mac-1. Il est probable que la fonction de PRR (pattern recognition receptor) de Mac-1, lui permette d'interagir avec d'autres bactéries pathogènes que L. monocytogenes [11] et que Mac-1 soit également impliqué dans l'activation de la LAP suite à l'infection par ces pathogènes. $\diamond$

The role of LC3-associated phagocytosis during infection by Listeria monocytogenes 
LIENS D'INTÉRÊT

Les auteurs déclarent n'avoir aucun lien d'intérêt concernant les données publiées dans cet article.

\section{RÉFÉRENCES}

1. Ray K, Marteyn B, Sansonetti PJ, Tang CM. Life on the inside the intracellular lifestyle of cytosolic bacteria. Nat Rev Microbiol 2009 ; 7 : 333-40.

2. Gluschko A, Herb M, Wiegmann K, et al The $\beta 2$ integrin Mac-l induces protective LC3associated phagocytosis of Listeria monocytogenes. Cell Host Microbe 2018 ; 23 : 324-337.e5.

3. Huang J, Canadien V, Lam GY, et al. Activation of antibacterial autophagy by NADPH oxidases. Proc Natl Acad Sci USA 2009; $106: 6226-31$.
4. Lam GY, Cemma M, Muise AM, et al. Host and bacterial factors that regulate $\mathrm{LC} 3$ recruitment to Listeria monocytogenes during the early stages of macrophage infection. Autophagy 2013; $9: 985-95$

5. Köster S, Upadhyay S, Chandra P, et al. Mycobacterium tuberculosis is protected from NADPH oxidase and LC3-associated phagocytosis by the LCP protein CpsA. Proc Natl Acad Sci USA 2017 ; 114 : ع8711-20.

6. Ehlers MRW. CR3: a general purpose adhesionrecognition receptor essential for innate immunity. Microbes Infect $2000 ; 2$ : 289-94.

7. Zhang Y, Li X, Carpinteiro A, Gulbins $\varepsilon$. Acid sphingomyelinase amplifies redox signaling in Pseudomonas aeruginosa-induced macrophage apoptosis. J Immunol 2008 ; 181: 4247-54.
8. Schramm M, Herz J, Haas A, et al. Acid sphingomyelinase is required for efficient phagolysosomal fusion. Cell Microbiol 2008 ; 10 : 1839-53.

9. Cerro-Vadillo E del, Madrazo-Toca F, CarrascoMarín $\varepsilon$, et al. Cutting edge: a novel nonoxidative phagosomal mechanism exerted by cathepsin-D controls Listeria monocytogenes intracellular growth. J Immunol 2006 ; 176 : 1321-5.

10. Boonhok R, Rachaphaew N, Duangmanee A, et al. LAP-like process as an immune mechanism downstream of IFN- $\gamma$ in control of the human malaria Plasmodium vivax liver stage. Proc Natl Acad Sci USA 2016 ; 113 : ع3519-28.

11. Podolnikova NP, Podolnikov AV, Haas TA, et al. Ligand recognition specificity of leukocyte integrin $\alpha M \beta 2$ (Mac-1, CDIlb/CD18) and its functional consequences. Biochemistry 2015 ; 54 : 1408-20.

\section{www.myobase.org}

Catalogue en ligne disponible gratuitement sur Internet publié par l'AFM-Téléthon.

Retrouvez facilement toutes les références bibliographiques sur les maladies neuromusculaires, les situations de handicap qu'elles génèrent et leurs aspects psychologiques.

Myobase donne un accès libre à $75 \%$ du fonds documentaire collecté depuis 1990 , représentant plus de 40000 références spécifiques du domaine des maladies neuromusculaires.

$>$ articles de la littérature biomédicale et psycho-sociale

\section{$>$ livres, thèses}

$>$ guides d'associations et rapports institutionnels d'agences internationales

$>$ brèves en français, synthèses des articles médico-scientifiques internationaux les plus pertinents

\section{$>$ publications}

AFM-Téléthon destinées aux professionnels de santé ou aux personnes atteintes de maladie neuromusculaire et à leur entourage

\section{UN OUTIL ERGONOMIQUE, UNE INTERFACE BILINGUE}

- Laissez-vous guider par les tutoriels

- Lancez une recherche et affinez votre sélection grâce aux filtres

TOUT MYOBASE
Rechercher...
Recherche avancee
\[ \text { Histo } \]
$\begin{array}{ll}\text { FILTRES } \\ \text { Type de document } \\ \square \text { Article [3443] } \\ \square \text { Publication AFM [176] } \\ \square \text { Thèse/Mémoire [107] } \\ \square \text { Brève [102] }\end{array}$

- PUBLICATIONS AFM-Téléthon

\section{• BRÈVES}

- DOCUMENTS DE SYNTHÈSE

\section{INSTITUT DES BIOTHÉRAPIES} PUBLICATIONS

- Partagez les résultats de votre recherche

\section{UN ACCÈS facile et simple}

Rechercher avec des opérateurs :

- guillemets pour une expression "maladie de pompe"

- + pour signifier ET, et retrouver tous les documents contenant les deux mots "fauteuil +électrique"

- - pour signifier NON et enlever le mot de la recherche:

"autonomie -établissement"
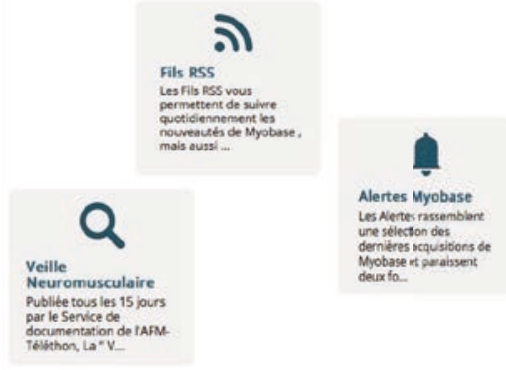

- Cliquez sur I'onglet

thématique qui vous convient (haut de la page d'accueil)

- Créez vos alertes personnalisées en ouvrant un compte personnel

- Téléchargez la Veille Neuromusculaire

- Abonnez-vous aux flux RSS 